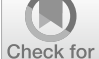

Check for

cosdates

Cite as

Nano-Micro Lett.

(2020) $12: 24$

Received: 21 October 2019

Accepted: 8 December 2019

Published online: 17 January 2020

(C) The Author(s) 2020

\section{Nitrogen-doped Carbon Nanospheres-Modified Graphitic Carbon Nitride with Outstanding Photocatalytic Activity}

\author{
Qiaoran Liu ${ }^{1}$, Hao Tian ${ }^{2}$, Zhenghua Dai ${ }^{3}$, Hongqi $\mathrm{Sun}^{4}$, Jian $\mathrm{Liu}^{2}, \mathrm{Zhimin} \mathrm{Ao}^{3}$, \\ Shaobin Wang ${ }^{5}$, Chen $\mathrm{Han}^{1}$, Shaomin $\mathrm{Liu}^{1}{ }^{凶}$ \\ \Shaobin Wang, Shaobin.wang@adelaide.edu.au; Shaomin Liu, Shaomin.Liu@curtin.edu.au \\ Department of Chemical Engineering, Curtin University, Perth, WA 6845, Australia \\ 2 State Key Laboratory of Catalysis, Dalian Institute of Chemical Physics, Chinese Academy of Sciences, \\ Dalian 116023, People's Republic of China \\ 3 School of Environmental Science and Engineering, Guangdong University of Technology, \\ Guangzhou 510006, People's Republic of China \\ 4 School of Engineering, Edith Cowan University, Joondalup, WA 6027, Australia \\ 5 School of Chemical Engineering, The University of Adelaide, Adelaide, SA 5005, Australia
}

\title{
HIGHLIGHTS
}

- Monodisperse nitrogen-doped carbon nanospheres were synthesized and loaded onto graphitic carbon nitride and the composites show outstanding photocatalytic activity.

- Improved sulfachloropyridazine degradation is consistent with density functional theory calculation..

\begin{abstract}
Metals and metal oxides are widely used as photo/ electro-catalysts for environmental remediation. However, there are many issues related to these metal-based catalysts for practical applications, such as high cost and detrimental environmental impact due to metal leaching. Carbon-based catalysts have the potential to overcome these limitations. In this study, monodisperse nitrogen-doped carbon nanospheres (NCs) were synthesized and loaded onto graphitic carbon nitride $\left(\mathrm{g}-\mathrm{C}_{3} \mathrm{~N}_{4}, \mathrm{GCN}\right)$ via a facile hydrothermal method for photocatalytic removal of sulfachloropyridazine (SCP). The prepared metal-free GCN-NC exhibited remarkably enhanced efficiency in SCP degradation. The nitrogen content in NC critically influences the physicochemical properties and performances of the resultant hybrids. The optimum nitrogen doping concentration was identified at $6.0 \mathrm{wt} \%$. The

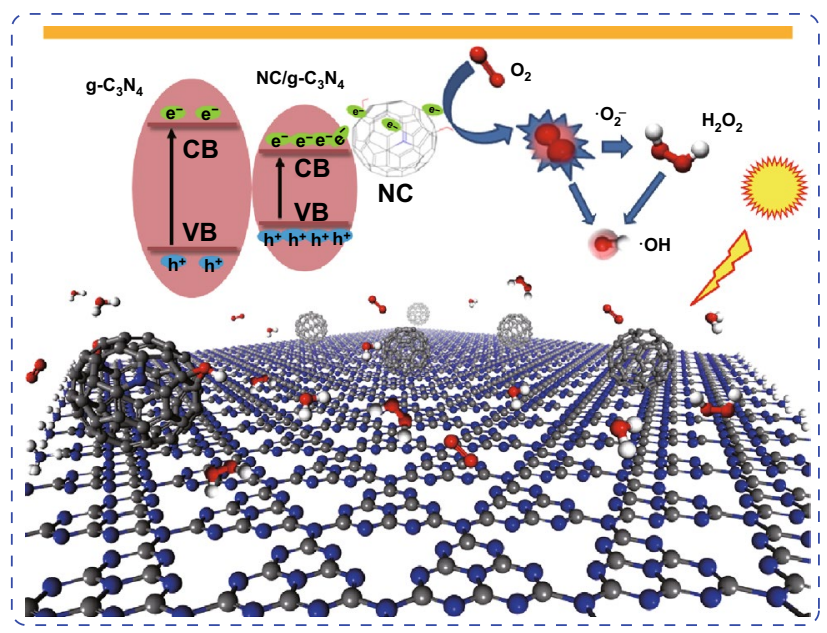
SCP removal rates can be improved by a factor of 4.7 and 3.2, under UV and visible lights, by the GCN-NC composite due to the enhanced charge mobility and visible light harvesting. The mechanism of the improved photocatalytic performance and band structure alternation were further investigated by density functional theory (DFT) calculations. The DFT results confirm the high capability of the GCN-NC hybrids to activate the electron-hole pairs by reducing the band gap energy and efficiently separating electron/hole pairs. Superoxide and hydroxyl radicals are subsequently produced, leading to the efficient SCP removal.
\end{abstract}

KEYWORDS N-doping; Carbon sphere; Graphitic carbon nitride; Photocatalysis; Degradation 


\section{Introduction}

In recent decades, human being has been enjoying the benefits of the technology development more than ever before in the human history. However, we are accompanied by sufferings from two issues: limited resources for raw materials and environmental pollution, one of which is water contamination. Some of the toxic pollutants are refractory to natural degradation; hence, it is crucial to take stringent measures to battle the situation [1-3]. Sulfachloropyridazine (SCP) is one of the sulphonamides widely used to be active against bacteria in aquaculture, animal husbandry, and human medicine. Such antibiotic exhibits high environmental mobility, presenting in both surface and ground waters. It is crucial to develop efficient technologies to remove recalcitrant SCP from polluted effluents. Currently, solar energy has been regarded as an abundant, clean and sustainable resource. Solar energy has attracted worldwide attention in many fields as an alternative supply to the conventional fossil fuel-based energy which produces the harmful emissions and impacts on human health and ecological systems [4]. For environmental remediation, metal-based catalysts like manganese [5], iron [6] or cobalt [7] have been applied, but they are costly and sometimes associated with metal leaching to secondary contamination [8]. Therefore, metal-free catalyst materials have been highly recommended as "green" photo- or electro-catalysts [3, 9]. It has been reported that carbon materials as metal-free sustainable catalysts, such as graphene [10], graphene oxide [1,10], carbon quantum dots [11], carbon nanotubes [12], and carbon nanospheres [9], can effectively promote photocatalytic reactions.

As a fascinating conjugated polymer, $\mathrm{g}_{-} \mathrm{C}_{3} \mathrm{~N}_{4}(\mathrm{GCN})$ has become a new research hotspot and drawn broad interdisciplinary attention in solar energy conversion because of the excellent stability to photocorrosion and chemical corrosion, exceptionally high thermodynamic stability, nontoxicity, and low band gap [13]. The polymer is composed of carbon and nitrogen, both of which are earth-abundant elements. Meanwhile, subsequent studies have shown that GCN-based photocatalysts are effective for various applications, such as contaminant decompositions [14, 15], hydrogen evolution reactions (HER) [16-18], oxygen evolution reactions (OER) [19], $\mathrm{CO}_{2}$ reduction [20], bioimaging and electricity generation. Yet, the low electrical conduction of the pristine GCN and the rapid photoelectron depletion cause the undesirable photon harvesting [21]. A variety of protocols involving heteroatom doping $[22,23]$, morphology modification [3, 24], hybrid copolymerization [25, 26], exfoliation [27] and co-catalysts have been employed to overcome these barriers and enhance the photocatalytic activity.

Among these methods, the modification by a carbon material [28] has become a hot focus, because of their potentials for enlarged specific area, good electronic conductivity and excellent rate of electron transfer [29-31]. In particular, our group has reported that size-tailored, uniformed carbon spheres can promote GCN toward enhanced photocatalysis [32]. Meanwhile, non-metal doping is demonstrated as an effective approach since the electronic structure and band gap would be changed dramatically via heteroatom doping [17, 33, 34]. Nitrogen-doped carbonaceous nanomaterials are found to be able to expedite the migration of photoinduced electron-hole pairs and promote the generation of active sites during the photocatalytic reaction by forming delocalized pi bonds [22, 35-38].

Herein, we integrate uniform nitrogen-doped carbon nanospheres (NC) with GCN to form NC/GCN composites via a one-step hydrothermal process. The mechanism of the enhancement of NC/GCN in terms of optical and electronic properties has been explored to remove SCP in aquatic environment. We also employed density functional theory (DFT) calculations [39] to investigate the GCN band gap energy $(\mathrm{Eg})$ variation with the introduction of carbon nanosphere or $\mathrm{N}$-doping. This work provides a facile way to modulate the intrinsic electronic and band structure of GCN and would lead to a simple and effective approach to synthesize an efficient NC/GCN photocatalyst via N-doping.

\section{Experimental}

\subsection{Materials}

Melamine powder ( $\geq 99.9 \%)$, resorcinol ( $\geq 99.0 \%)$, formaldehyde (37\% solution), 3-aminophenol ( $\geq 99 \%)$, 4-amino3-nitrophenol ( $\geq 99 \%$ ), aqueous ammonia (25\% solution) and ethanol ( $\geq 99.9 \%)$ were provided by Sigma-Aldrich and used as received without any further purification. 


\subsection{Synthesis of Graphitic Carbon Nitride}

A conventional condensation method was employed to prepare carbon nitride utilizing melamine as the precursor. In a typical process, $10 \mathrm{~g}$ of melamine and $10 \mathrm{~mL}$ of methanol were added into a $20-\mathrm{mL}$ crucible and well mixed by stirring. The crucible with a cover was then placed in an oven at $60{ }^{\circ} \mathrm{C}$ for $24 \mathrm{~h}$ to remove the methanol. Followed by transferring into a muffle furnace, the crucible was firstly heated up to $400{ }^{\circ} \mathrm{C}$ at a heating rate of $5{ }^{\circ} \mathrm{C} \mathrm{min}^{-1}$ and kept at this temperature for $2 \mathrm{~h}$. Subsequently, the furnace was heated to $520^{\circ} \mathrm{C}$ at a rate of $10{ }^{\circ} \mathrm{C} \mathrm{min}^{-1}$ and kept at this temperature for another $2 \mathrm{~h}$. When it was cooled down to room temperature, the resulting yellow powder was ground and denoted as GCN for further use.

\subsection{Synthesis of Carbon Nanospheres and Nitrogen-doped Carbon Nanospheres}

Monodisperse NCs with diameter around $400 \mathrm{~nm}$ were synthesized. In a typical run, $0.5 \mathrm{~mL}$ of ammonia aqueous solution $\left(\mathrm{NH}_{3} \mathrm{H}_{2} \mathrm{O}\right)$ was added to $100 \mathrm{~mL}$ of ethanol solution (volume ratio of ethanol and distilled water was 2:5) at room temperature. Then, $2.0 \mathrm{~g}$ of 3-aminophenol was added to the solution under stirring for $0.5 \mathrm{~h}$. Afterward, $2.8 \mathrm{~mL}$ of $37 \mathrm{wt} \%$ formaldehyde was added dropwise continuously and kept stirring for $24 \mathrm{~h}$. The obtained mixture was heated in an oven at $100^{\circ} \mathrm{C}$ for $24 \mathrm{~h}$. The carbonation process was completed in a tube furnace using a rate of $1{ }^{\circ} \mathrm{C} \mathrm{min}-1$ up to $350{ }^{\circ} \mathrm{C}$ under $\mathrm{N}_{2}$ flowing and dwelling for $2 \mathrm{~h}$. After this, the temperature was increased to $700{ }^{\circ} \mathrm{C}$ at a rate of $1{ }^{\circ} \mathrm{C} \min ^{-1}$ and kept for $4 \mathrm{~h}$ to obtain the black powder and labeled as NC (5.4). As shown in Table 1, the nitrogen content of this sample was $5.4 \%$ by elemental analysis. During the preparation of $\mathrm{NC}$, the volume proportion of ethanol and water was changed to 1:6, and a NC sample with nitrogen content of $6.0 \mathrm{wt} \%$ was obtained and denoted as NC (6.0). Similarly, NC (11.9) was synthesized by changing 3-aminophenol to 4-amino-3-nitrophenol. Control carbon nanospheres with a similar size were prepared by the method reported by Liu et al. [7] and labeled as C.

\subsection{Preparation of GCN-NC and GCN-C}

The GCN-NC and GCN-C hybrid photocatalysts were synthesized by a hydrothermal treatment using the prepared GCN-NC and GCN-C as the precursors. Firstly, $0.05 \mathrm{~g}$ of
Table 1 Elemental analysis (weight percentage) of the nitrogendoped carbon nanospheres

\begin{tabular}{llcll}
\hline Sample & $\mathrm{C} \%$ & $\mathrm{~N} \%$ & $\mathrm{H} \%$ & $\mathrm{O} \%$ \\
\hline GCN & 41.18 & 58.82 & - & - \\
C & 83.92 & \multicolumn{1}{c}{-} & - & 16.08 \\
NC(5.4) & 77.18 & 5.43 & 1.71 & 15.68 \\
NC(6.0) & 76.74 & 6.02 & 1.70 & 15.54 \\
NC(11.9) & 72.45 & 11.90 & 1.52 & 14.13 \\
\hline
\end{tabular}

$\mathrm{NC}$ or $\mathrm{C}$ spheres together with $0.95 \mathrm{~g}$ of pure $\mathrm{GCN}$ were put into $50 \mathrm{~mL}$ of water and stirred for $3 \mathrm{~h}$ with subsequent ultrasonication for $4 \mathrm{~h}$ at room temperature to generate wellmixed suspensions. Secondly, the suspension was transferred into the 100-mL Teflon-lined autoclaves which were then heated at $150{ }^{\circ} \mathrm{C}$ for $12 \mathrm{~h}$. The autoclaves were cooled down to room temperature, and the obtained solids were washed thoroughly with ethanol and deionized water. After drying at $80{ }^{\circ} \mathrm{C}$ overnight, the obtained samples were denoted as GCN-NC(5.4), GCN-NC(6.0), GCN-NC(11.9), and GCN$\mathrm{C}$, respectively.

\subsection{Characterizations}

The crystal structure of the prepared samples was investigated by X-ray diffraction (D/Max-2500, Rigaku Corp) with a $\mathrm{Cu} K \alpha$ radiation $(\lambda=1.5406 \mathrm{~A})$ at a scan rate of $0.05 \%$. The Brunauer-Emmett-Teller (BET) surface areas and the pore size distributions of the samples were measured by an automated gas adsorption-desorption analyzer (TriStar II Plus, Micromeritics, USA). Field emission scanning electron microscopy (FESEM) images of the samples were taken on Zeiss Neon 40EsB to analyze the morphology, size and textural properties of the as-prepared samples. The functional groups of the samples were evaluated by a PerkinElmer Fourier transform infrared spectroscopy (FTIR)-100 with a MIR detector. The surface chemical states were obtained by an $\mathrm{X}$-ray photoelectron spectrometer on a Thermo Escalab 250 with an $\mathrm{Al} \mathrm{K} \alpha \mathrm{X}$-ray radiation source. The light absorption properties of photocatalysts were collected on a UV-visible diffuse reflectance spectra (DRS) spectrophotometer (JASCO V670) with an $\varnothing 60 \mathrm{~mm}$ integrating sphere and $\mathrm{BaSO}_{4}$ as a reference material. Photoluminescence (PL) spectra were measured using a Hitachi F-4500 fluorescence spectrophotometer at room temperature. Electron 
paramagnetic resonance (EPR) experiments were performed on a Bruker EMXplus spectrometer (Germany) to analyze the generation of active radicals during the photocatalytic reactions. Electrochemical experiments were conducted on a Zennium electrochemical workstation in a conventional three-electrode cell with $0.2 \mathrm{M} \mathrm{Na}_{2} \mathrm{SO}_{4}$ solution as electrolyte, $\mathrm{Hg} / \mathrm{Hg}_{2} \mathrm{Cl}_{2}$ as the reference electrode, and a platinum wire as the auxiliary electrode. Four on-off cycles were performed by turning on/off the light for $30 \mathrm{~s}$. Electrochemical impedance spectroscopy (EIS) was obtained under an opencircuited potential with the signal amplitude of $5 \mathrm{mV}$ and frequency ranges from $10^{6}$ to $10^{-1} \mathrm{~Hz}$.

\subsection{Photocatalytic Activity Testing}

Photodegradation tests were carried out in a conical flask containing $200 \mathrm{~mL}$ ( $30 \mathrm{mg} \mathrm{L}^{-1}$ ) of SCP solution at $25^{\circ} \mathrm{C}$ under CEL-LAX Xe lamp irradiations (UV cutoff filter $<400 \mathrm{~nm}$ and visible light cutoff filter $400-700 \mathrm{~nm}$ ). The photograph of the photocatalysis setup is shown in Fig. S3. The reaction system was placed $30 \mathrm{~cm}$ away from the light source. Firstly, $100 \mathrm{mg}$ of photocatalyst was placed inside SCP solution in a double-jacket cylindrical reactor with water cycling and constantly stirring at $400 \mathrm{rpm}$ for $30 \mathrm{~min}$ to obtain the adsorption-desorption equilibrium. After that, the photocatalytic degradation was triggered by switching on the light source. At regular intervals, $1 \mathrm{~mL}$ sample aliquot was withdrawn and passed through a $5-\mathrm{mL}$ syringe filter unit ( $0.45 \mu \mathrm{m}$ pore) for analysis by high-performance liquid chromatography (HPLC). The concentration was analyzed using the HPLC with an UV detector at wavelength of $270 \mathrm{~nm}$. In the multi-cycle run experiment, the photocatalytic performance of composite catalyst was proceeded continuously without catalyst recovery.

\subsection{DFT Calculations}

The core electrons were treated by the ultra-soft pseudopotential, and the exchange and correlation effects were described by the Perdew-Burke-Ernzerhof (PBE) within generalized gradient approximation (GGA) [40]. In order to accurately describe the nonbonding van der Waals interaction along c-axis, the DFT + D method within the TS scheme was used in all calculations [41].

\section{Results and Discussion}

\subsection{Characterization of Prepared Samples}

SEM images reveal the morphologies of the four GCNbased composites. As presented in Fig. 1a-d, the GCNbased composites show a typical lamellar morphology, and $5 \mathrm{wt} \%$ nitrogen-doped carbon nanospheres are closely attached onto the surface of layered GCN homogeneously. $\mathrm{NC}$ spheres with uniform diameters around $400 \mathrm{~nm}$ are found in GCN-NC(5.4), GCN-NC(11.9) and GCN-NC(6.0) composites (Fig. 1b-d). From TEM images (Fig. 1e, f), we can see these carbon nanospheres can be immobilized onto GCN, and the size of carbon sphere was $400 \mathrm{~nm}$, which is a suitable size to form strong interaction with GCN as observed previously [32]. XRD analysis (Fig. 1g) was performed to investigate the phase structure of the pristine GCN and hybrid GCN composite samples. The pristine GCN sample exhibits two characteristic reflections at $13.1^{\circ}$ and $27.6^{\circ}$ corresponding to the diffraction planes of (002) and (100), respectively [12]. The two peaks were found to be in good agreement with reported results, illustrating the successful synthesis of GCN with interplanar stacking fused aromatic rings and in-planar structure of triazine units. The main diffraction intensities of the GCN-NC composites are not substantially changed with the increase in nitrogen content in NC. It can be seen from Fig. S1 that the FTIR spectra of pure GCN and carbon sphere-loaded GCN composites show no noticeable difference. Peaks in the region of $3000-3500 \mathrm{~cm}^{-1}$ originate from the adsorption of $\mathrm{H}_{2} \mathrm{O}$ and $\mathrm{CO}_{2}$ in the air. Several strong bands from 1200 to $1700 \mathrm{~cm}^{-1}$ are found in the samples, and the peaks at 1570 and $1630 \mathrm{~cm}^{-1}$ correspond to the $\mathrm{C}=\mathrm{N}$ stretching [42]. The detection of characteristic peaks of the GCN indicates that the GCN was successfully formed via thermal polymerization. $\mathrm{N}_{2}$ adsorption and desorption isotherms of pristine GCN and GCN-based composites are elucidated in Fig. 1h, which mirrors a type-IV adsorption behavior. The hysteresis loops are $\mathrm{H} 3$ type with a mesoporous structure. GCN-NC(11.9) exhibited the largest surface area and pore volume, as illustrated in the inset table. The BET surface area of GNC-NC (6.0) was almost 5 times higher than GCN (33.1 vs $7.1 \mathrm{~m}^{2} \mathrm{~g}^{-1}$ ); thus, GNC-NC(6.0) can provide more active sites to promote the adsorption of reactant, favorable for catalytic reaction [43]. 

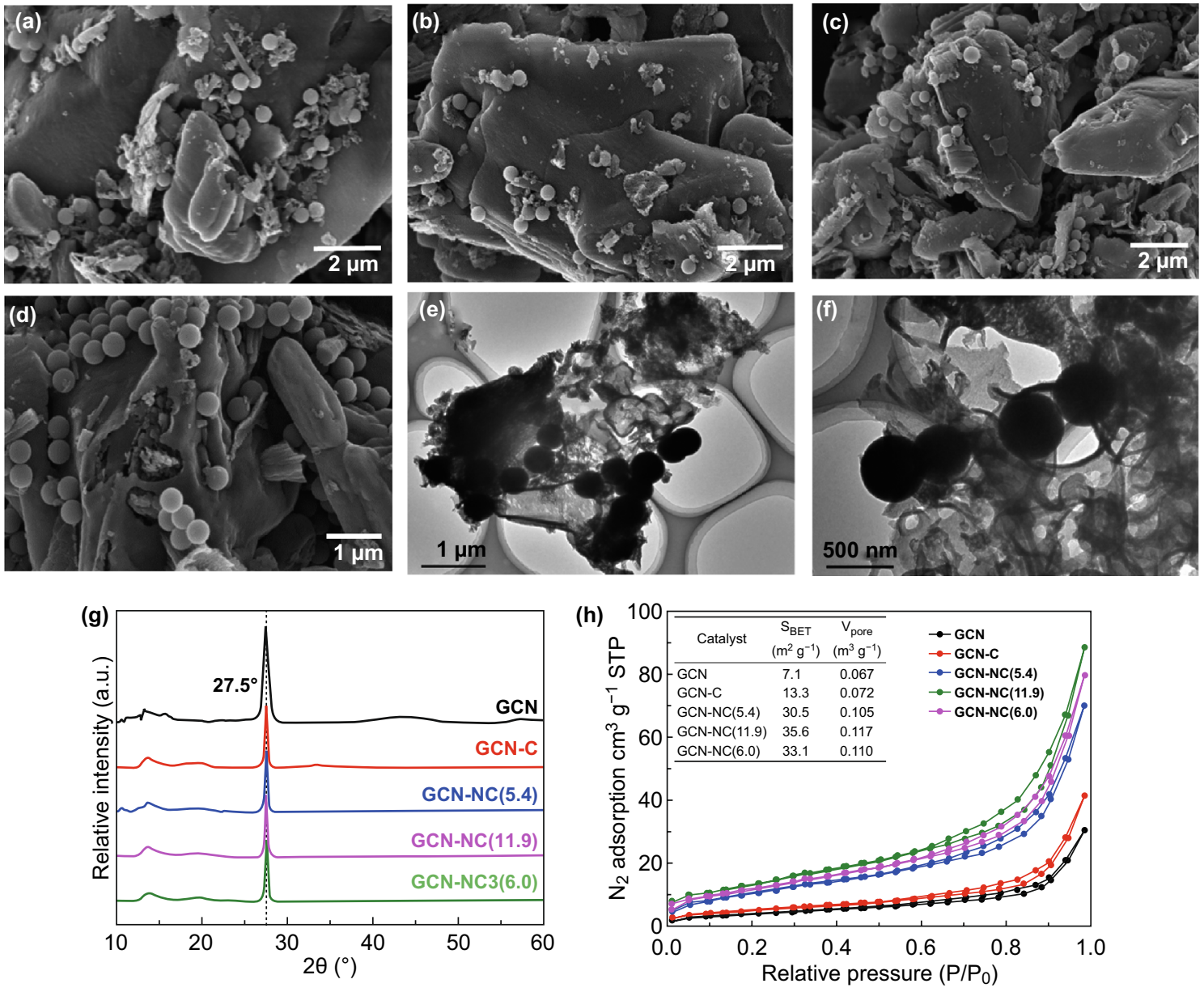

Fig. 1 a SEM images of GCN-C, b GCN-NC(5.4), $\mathbf{c}$ GCN-NC(11.9), and d GCN-NC(6.0). e, $\mathbf{f}$ TEM images of GCN-NC(6.0). $\mathbf{g}$ XRD patterns; h $\mathrm{N}_{2}$ adsorption-desorption isotherms (h inset: the textural properties) of samples

The chemical composition of GCN-NC nanocomposites was further investigated by X-ray photoelectron spectroscopy (XPS) in the region from 0 to $800 \mathrm{eV}$. The full survey shows two typical peaks for GCN with the corresponding content of $56.62 \%$ and $43.38 \%$ for $\mathrm{N} 1 s$ and C $1 s$ (Fig. 2a), respectively. The surface oxygen contents of the compounds are relatively low from $1.80 \%$ to $2.83 \%$, possibly stemmed from the partial oxidation by $\mathrm{O}_{2}$ and $\mathrm{H}_{2} \mathrm{O}$ during the hydrothermal process. The high-resolution narrow XPS scan spectra show two overlapping $\mathrm{C} 1 s$ peaks corresponding to the $s p^{2}$ bonded carbon $(\mathrm{C}-\mathrm{C} / \mathrm{C}=\mathrm{C})$ and the carbon adjacent with three $\mathrm{N}$ atoms $(\mathrm{C}-\mathrm{C}=\mathrm{N})$ [44]. There was a small peak of $s p^{2}$ bonded carbon in pristine GCN since it is common to have adsorbed graphitic or amorphous carbon on the surface. However, this peak was stronger when carbon nanospheres were loaded onto GCN and the density of which decreased with the nitrogen content in carbon nanospheres. The position shift of $\mathrm{C} 1 s$ peak in these samples also implies the interaction between GCN and carbon nanosphere. Based on peak deconvolution, the ratio of $\mathrm{N} 1 s$ spectrum in the composite is described in Fig. S2 and the relative amount of different nitrogen groups is summarized in Table 2. The $\mathrm{N}$ $1 s$ XPS spectrum is deconvoluted to four peaks. The one at $398.6 \mathrm{eV}$ could be assigned to the $s p^{2}$ hybridized $\mathrm{N}$ bonded with two carbon atoms $(\mathrm{C}-\mathrm{N}=\mathrm{C})$ and the dominant nitrogen species in the GCN with tris-triazine structure with the carbon. Spectrum at $399.5 \mathrm{eV}$ can be assigned to the tertiary $\mathrm{N}$ ( $\mathrm{N}$ bonded with three carbon atoms, $\left.\mathrm{N}-(\mathrm{C})_{3}\right)$. The other two peaks at 400.8 and $397.7 \mathrm{eV}$ correspond to the amino groups (C-NH) and N-graphene.

In Fig. 3a, the optical absorption abilities of pristine GCN and NC-modified GCN samples were analyzed 

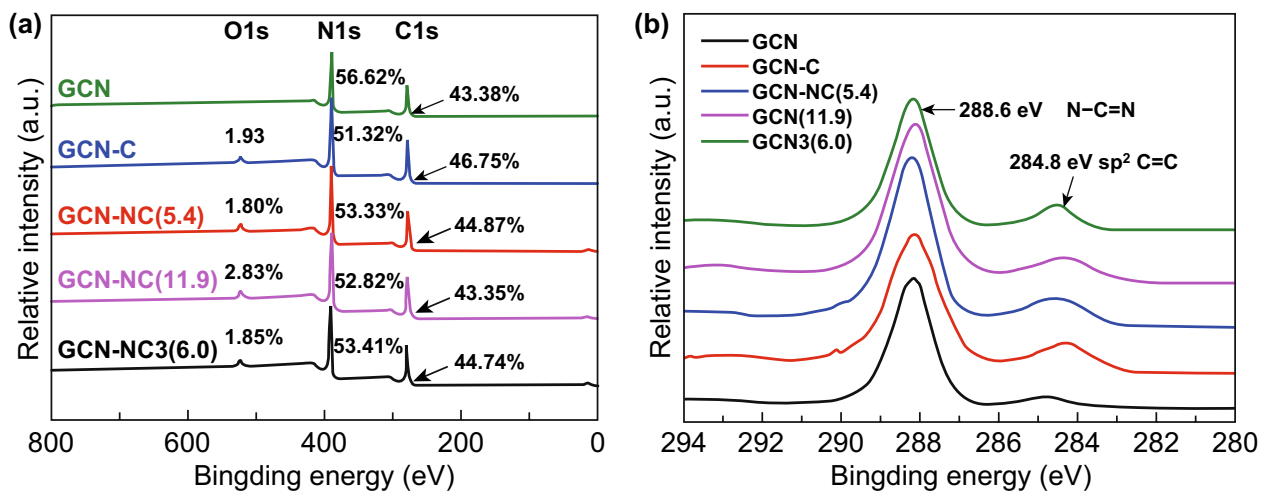

Fig. 2 a XPS full survey and b C $1 s$ spectra of GCN, GCN-C, GCN-NC(5.4), GCN-NC(11.9), and GCN-NC(6.0)

Table 2 Total content and relative amount of different nitrogen groups on the surface of the catalysts

\begin{tabular}{llllll}
\hline Sample & $\begin{array}{l}\text { N content on } \\
\text { the surface } \\
\text { (at\%) }\end{array}$ & $\mathrm{C}-\mathrm{N}=\mathrm{C}$ & $\mathrm{N}-(\mathrm{C})_{3}$ & $\mathrm{C}-\mathrm{NH}$ & $\mathrm{N}$-graphene \\
\hline $\mathrm{GCN}$ & 56.62 & 60.21 & 17.67 & 10.69 & 11.44 \\
$\mathrm{GCN}-\mathrm{C}$ & 51.32 & 50.26 & 24.27 & 15.55 & 9.91 \\
$\mathrm{GCN}-$ & 53.33 & 48.82 & 22.23 & 13.71 & 15.25 \\
$\mathrm{NC}(5.4)$ & & & & & \\
$\mathrm{GCN}-$ & 52.82 & 49.66 & 20.07 & 12.86 & 17.41 \\
$\mathrm{NC}(11.9)$ & & & & & \\
$\mathrm{GCN}-$ & 53.41 & 42.29 & 21.11 & 22.29 & 13.31 \\
$\mathrm{NC}(6.0)$ & & & & & \\
\hline
\end{tabular}

using UV-Vis absorption spectroscopy. The GCN sample shows a typical feature of photosensitive semiconductor with an intrinsic characteristic absorption peak at
$420 \mathrm{~nm}$, corresponding to a band gap energy of $2.46 \mathrm{eV}$ for photoexcited electron, which is calculated based on the Kubelka-Munk formula as shown in the inset of Fig. 3a. Such observation is attributed to the lone pair of electrons of nitrogen atom in valance band jumping into the $\pi$ bonding electronic conduction band. The redshift derived from hybridization can be observed, and the detected absorption thresholds of GCN-C, GCN-NC(5.4), GCN-NC(11.9), and GCN-NC(6.0) were 463, 487, 491, and $520 \mathrm{~nm}$, respectively. The respective band gap energies of these samples were evaluated to be $2.29,2.17,2.12$, and $2.08 \mathrm{eV}$, which indeed reflect the changes at the interface with the integration of carbon spheres. Compared with GCN, carbon sphere and nitrogen-doped carbon sphere have better visible light adsorption. Therefore, the absorptive capability of all composite materials was improved in the entire visible light
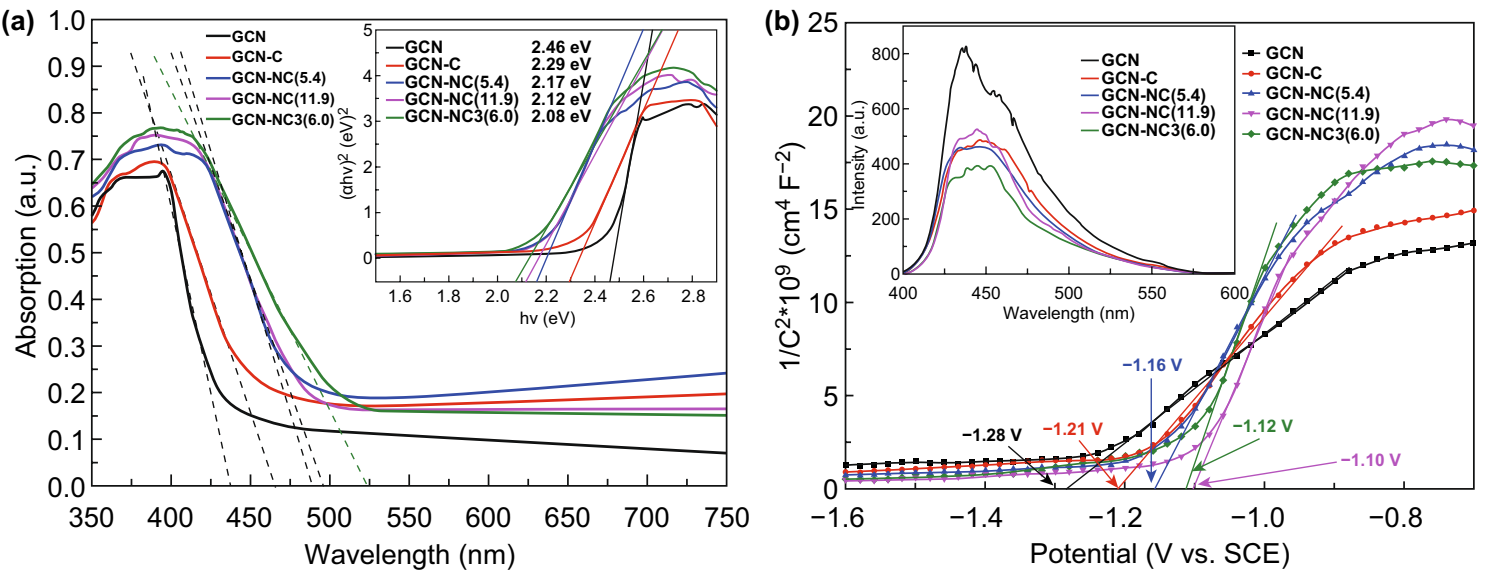

Fig. 3 a UV-Vis diffuse reflectance absorption and b Mott-Schottky of GCN and NC-loaded GCN. The inset in a shows plots of ( $\alpha$ h $\nu)^{2}$ versus photon energy (hv) for the band gap energy; the inset in b shows the photoluminescence spectra of the samples 
region compared to pure GCN, especially for GCN-NC(6.0). The electrochemical Mott-Schottky plots were acquired to determine the energy levels, which features a typical n-type semiconductor in Fig. 3b. The conduction band potentials (CB) of GCN, GCN-C, GCN-NC(5.4), GCN-NC(11.9), and GCN-NC(6.0) were estimated to be $-1.28,-1.21$, $-1.16,-1.10$, and $-1.12 \mathrm{eV}$, respectively. Accordingly, the valence band positions (VB) were then estimated according to Eq. 1:

$E_{g}=E_{\mathrm{VC}}-E_{\mathrm{VB}}$

The band structures of the samples are compared in Scheme 1. It can be seen that the introduction of NC onto GCN resulted in a negatively shift of VB position. The photodegradation efficiency can be partially determined by the separation rate of the photoinduced carriers on the composite materials. The accelerated charge carrier transfer of bare GCN, GCN-C, GCN-NC(5.4), GCN-NC(11.9), and GCN-NC(6.0) was exhibited through the PL emission (inset in Fig. 3b) under $320 \mathrm{~nm}$ exciting radiations. From 400 to $550 \mathrm{~nm}$, the single luminescence peak of GCN was stronger than the hybrid photocatalysts, indicating that the composite structure provided a synergistic effect for GCN to separate the photoexcited carriers from the valance band. In particular, the recombination rate of photoinduced charges of GCN-NC(6.0) decreases more significantly, whereas the electron-hole recombination of GCN-C is slightly higher. In addition, when the exciting light was irradiated on the GCNNC composites, the PL spectrum has a slight dislocation to $450 \mathrm{~nm}$ compared with that of the pure GCN sample at $440 \mathrm{~nm}$, which might further signal the interaction between GCN and NC.
The adsorption and photodegradation of SCP were carried out to evaluate the remediation performance of the prepared samples. Figure $\mathrm{S} 4$ shows control results of SCP adsorption on GCN-NC(6.0) and photolysis of SCP without catalyst. Only $8.2 \%$ of SCP were removed due to physical adsorption on GCN-NC(6.0), and SCP could hardly be degraded under both UV and visible light without catalyst presence. The photocatalytic degradation results under UV light irradiations are shown in Fig. 4a. It is observed that the composite photocatalysts had a higher adsorption rate than GCN in dark. GCN-NC(11.9) showed the best adsorption, which might be due to the largest specific surface area as revealed by BET analysis. As can be seen, SCP degradation reached $84 \%$ within 180 min by employing GCN, while the degradation efficiency of GCN-C was up to $100 \%$ in $150 \mathrm{~min}$. GCN-NC(5.4) and GCN-NC(11.9) achieved complete degradation in $90 \mathrm{~min}$. All of SCP was degraded by GCN-NC(6.0) within $60 \mathrm{~min}$, manifesting that the hybrid photocatalysts can provide the best performance. The reaction rate constant of GCN-NC(6.0) was calculated to be $0.0205 \mathrm{~min}^{-1}, 4.36$ times higher than that of $\mathrm{GCN}\left(0.0047 \mathrm{~min}^{-1}\right)$. The reaction rate constants of GCN-C, GCN-NC(5.4) and GCN-NC(11.9) were $0.0076,0.0154$, and $0.0139 \mathrm{mg} \mathrm{min}^{-1}$, respectively, under UV light irradiation (Fig. 4c) based on the following pseudo-first-order kinetics (Eq. 2):

$\ln \left(C_{0} / C\right)=-k t$

where $C_{0}$ is the concentration of the pollutant at the beginning of the reaction, $C$ is the pollutant concentration at the time $(t)$, and $k$ is the reaction rate constant. The calculated values of $k$ and $R^{2}$ are listed in Table. S1. It is found in Fig. $4 \mathrm{~b}$ and Table $\mathrm{S} 1$ that the photocatalysts demonstrated a similar trend under visible light irradiations. GCN-NC(6.0)

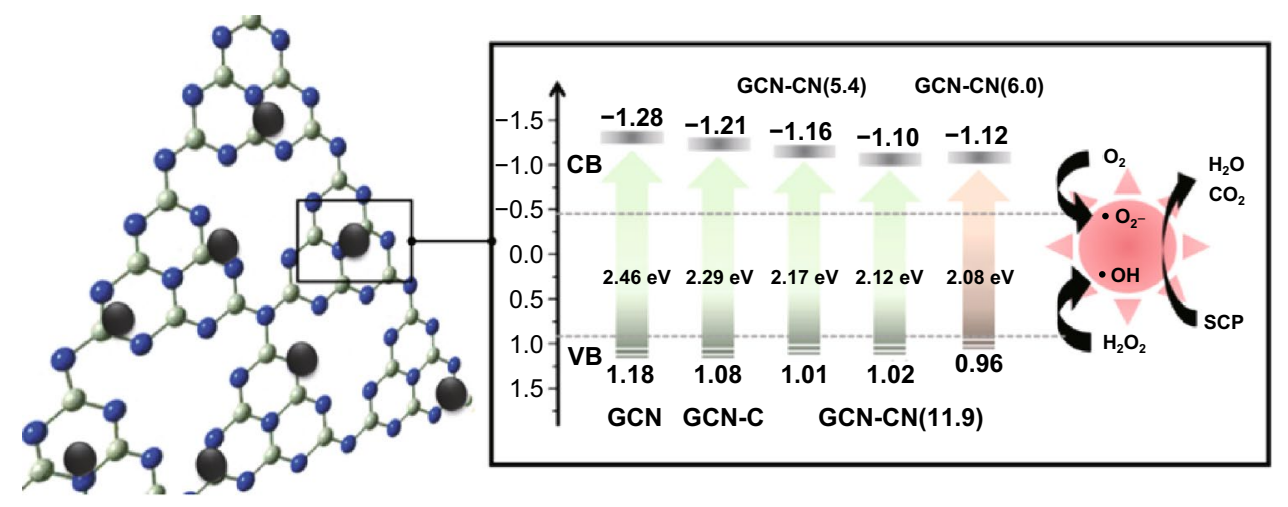

Scheme 1 Proposed mechanism for photocatalytic process 

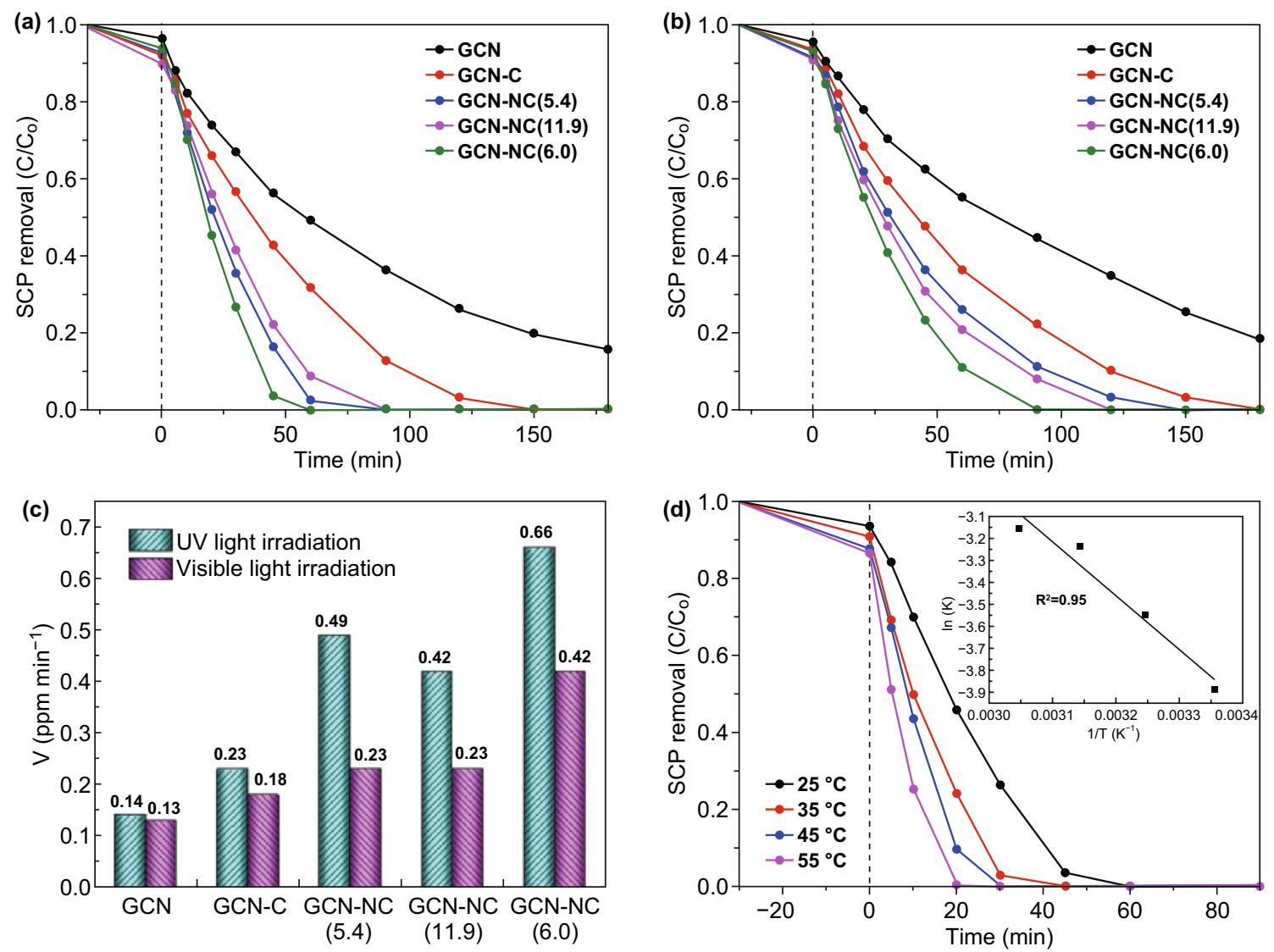

Fig. 4 a Photocatalytic degradation of SCP over GCN, GCN-C, GCN-NC(5.4), GCN-NC(11.9), and GCN-NC(6.0) under UV light irradiation and $\mathbf{b}$ under visible light irradiation. $\mathbf{c}$ Comparison of the degradation rate constants of GCN, GCN-C, GCN-NC(5.4), GCN-NC(11.9), and GCN-NC(6.0) photocatalysts under UV and visible light irradiations. $d$ The effect of temperature on degradation over GCN-NC(6.0)

showed the highest photodegradation efficiency with complete SCP removal in $90 \mathrm{~min}$, favorable to GCN-NC(11.9), GCN-NC(5.4), and GCN-C of 120, 150, and $180 \mathrm{~min}$, respectively, while the pristine GCN exhibited a poor activity with $18 \%$ of SCP remaining after $180 \mathrm{~min}$. And the degradation rates were also fitted by the first-order kinetics. GCN-NC(6.0) presented the best photodegradation with the reaction rate constants of $0.0139 \mathrm{~min}^{-1}$, which is about 3.3 times higher than GCN $\left(0.0042 \mathrm{~min}^{-1}\right)$. The reaction rate constants of GCN-C, GCN-NC(5.4), and GCN-NC(11.9) were $0.0061,0.0077$, and $0.0078 \mathrm{~min}^{-1}$, respectively. The observed less efficiency of GCN-C than GCN-NC in SCP photodegradation in Fig. 4a, b, illustrates that the nitrogendoped carbons are more favorable for charge transferring from GCN to adjacent carbon atoms, giving rise to positively charged sites (holes) to improve the photodegradation efficiency. It is interesting to note that the nitrogen content would impact the catalytic performance in the photocatalytic reaction, but the catalyst with extra high $\mathrm{N}$ content would limit visible light absorption and reduce the conductivity, resulting in a lowered activity. Combining the elemental analysis and XPS in Tables 1 and 2, the solar activation did not show the same trend with the nitrogen content of the carbon spheres. The one with nitrogen content of $6.0 \%$ is more efficient than the others with $11.9 \%$ and $5.4 \%$. However, the nitrogen should be at an appropriate level; otherwise, too higher level of nitrogen would result in a lower degradation. SCP degradation within a temperature range of $35-55^{\circ} \mathrm{C}$ was examined for $\mathrm{GCN}-\mathrm{NC}(6.0)$ photocatalyst (Fig. 4d). An enhanced trend can be observed with temperature elevation. GCN-NC(6.0) provided $100 \%$ SCP removal in $60,45,30$, and $20 \mathrm{~min}$ when the reaction took place at $25,35,45$, and $55^{\circ} \mathrm{C}$, respectively. The results suggest an endothermic reaction in this catalytic process. The kinetic rate constants are presented in Table S1. The ln k against 1/T plotted in Fig. 4d follows the Arrhenius equation, and the obtained activation energy was $20.32 \mathrm{~kJ} \mathrm{~mol}^{-1}$. Additionally, stability tests of the optimized photocatalyst were performed with result shown in Fig. S5. SCP was decomposed in 90 min for the first run under visible light. For the 
subsequent two runs, GCN-NC(6.0) achieved $100 \%$ of SCP removal in 120 and $150 \mathrm{~min}$. In the fourth run, only $92 \%$ degradation was achieved (Fig. S5a). A decreasing trend of degradation efficiency under UV light irradiation was also observed (Fig. S5b). As seen in Fig. S6, the deposition of the intermediates on the surface of catalysts resulted in the blockage of surface reactive sites. The deactivation of the photocatalysts could be ascribed to the alteration of surface charges and the detachment of nitrogen-doped carbon nanospheres during the photocatalysis processes. The samples after recycle experiments were recharacterized by XRD and UV-Vis measurements (Fig. S7). The results indicate that the morphology and optical property of GCN-NC(6.0) were not altered significantly after four cycles. Thus, the catalyst could be reused in practical pollution treatment. The correlation between $\mathrm{pH}$ and photodegradation of antibiotics by GCN has been well documented by previous studies [45, 46]. The increase in $\mathrm{pH}$ value lowers the proportion of neutral SCP, but enlarges the proportion of negatively charged species, thus justifying an overall acceleration in direct photolysis. On the other hand, the $\mathrm{pH}$ value increase can also lead to the promoted indirect photolysis, favoring SCP oxidation due to the generation of highly reactive radicals.

In Fig. 5a, a comparison of the on/off photocurrent response of nitrogen-doped carbon-decorated GCN under visible light irradiations was studied. The photocurrent ascended until it reached a constant value with light on and then declined with light off, suggesting that these GCNbased samples were light-responsive materials. GCN produced a stable density of $1.8 \mu \mathrm{A} \mathrm{cm} \mathrm{cm}^{-2}$ photocurrent, while all of the GCN-C, GCN-NC(5.4), and GCN-NC(11.9) enlarged the photocurrents to around $2.0 \mu \mathrm{A} \mathrm{cm}{ }^{-2}$. GCN$\mathrm{NC}(6.0)$ produced the biggest gap of photocurrent densities with the periodic irradiations, indicating that the lifetime of photogenerated carriers significantly increased [47]. Initially, GCN-NC(6.0) had a photocurrent of $2.7 \mu \mathrm{A} \mathrm{cm}{ }^{-2}$,
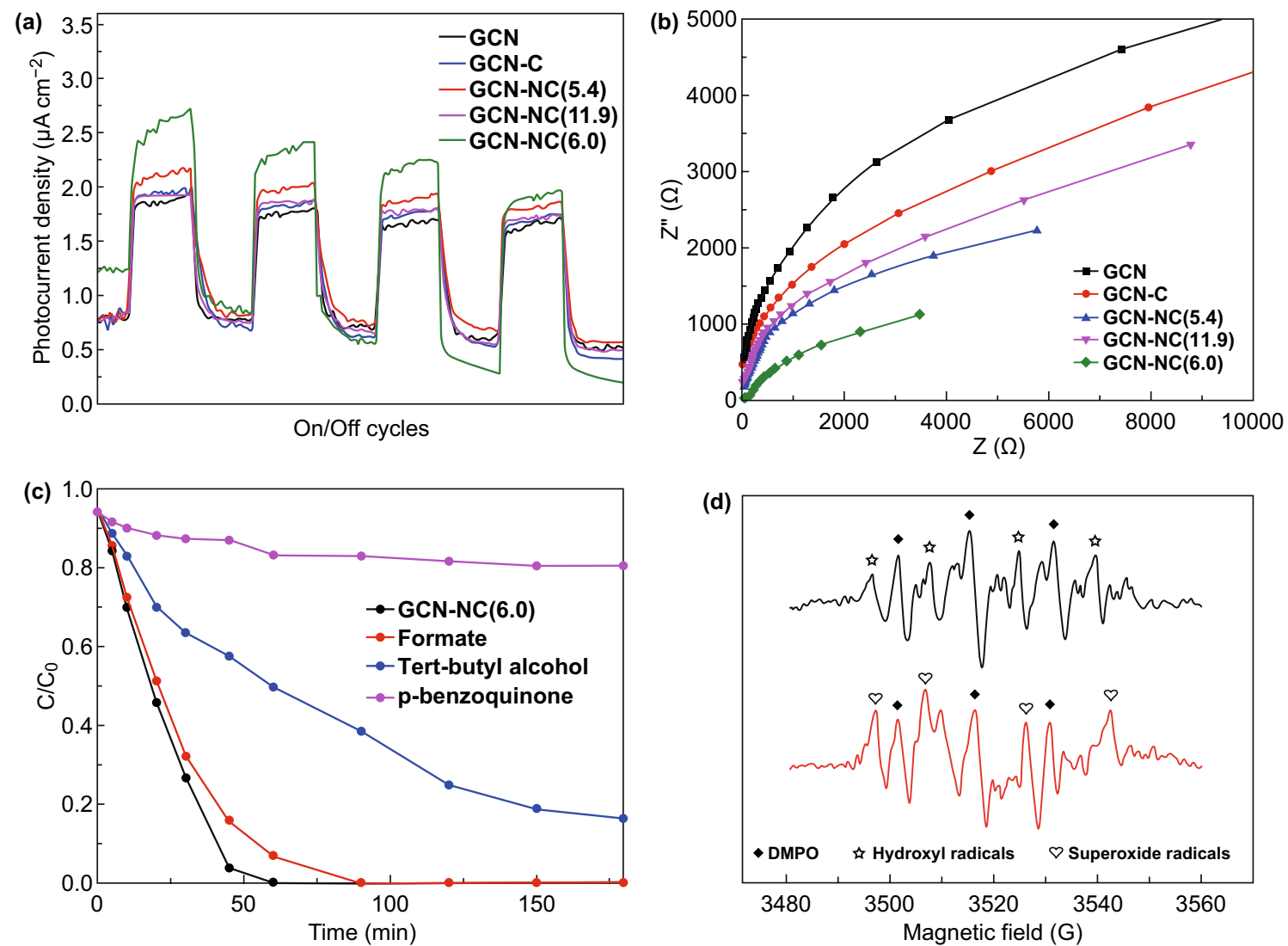

Fig. 5 a Transient photocurrent and b EIS curves of the GCN, GCN-C, GCN-NC(5.4), GCN-NC(11.9), and GCN-NC(6.0) in 0.1 M Na $2 \mathrm{SO}_{4}$ solution. c Photocatalytic activity of GCN-NC(6.0) for SCP degradation with radical scavengers (formate, tert-butyl alcohol, and p-benzoquinone) under visible light irradiations. d EPR spectra of GCN-NC(6.0) under UV-visible light irradiations with quenching of tert-butyl alcohol and p-benzoquinone 
but it decreased to $2.0 \mu \mathrm{A} \mathrm{cm}{ }^{-2}$ at the fourth cycle. This distinguishable enhancement of photocurrent response reveals that introducing nitrogen-doped carbon spheres can accelerate the transport of the photoinduced carriers and the separation of photoexcited electron/hole pairs. To make further verification of the electrochemical properties, the EIS Nyquist plots of the pristine GCN and nitrogendoped carbon-loaded GCN under visible light irradiations are recorded in Fig. 5b. The smallest radius implied the fastest interfacial electron transfer. The Nyquist plot of GCN-NC(6.0) displayed a smaller arc radius EIS than other samples, suggesting that it possesses the lowest resistant barrier to electron transfer. All these results demonstrate that $\mathrm{NC}(6.0)$ can function as an efficient co-catalyst in the GCN-NC(6.0) composite to effectively reduce the charge recombination that favors enhanced photocatalytic activity, which is in accordance with the PL spectra. Radical trapping experiments were carried out to differentiate the reactive oxygen species that play the key role in the photocatalytic process. Three radical scavengers, formate, tert-butyl alcohol, and p-benzoquinone, were employed to quench photogenerated holes $\left(\mathrm{h}^{+}\right)$, hydroxyl radicals $(\cdot \mathrm{OH})$, and superoxide radical $\left(\cdot \mathrm{O}_{2}^{-}\right)$, respectively. Figure $5 \mathrm{c}$ illustrates that the removal rates of SCP were remarkably inhibited after the addition of quenchers and the decline rates follow an order of p-benzoquinone $>$ tert-butyl alcohol $>$ formate, testifying that photogenerated $\cdot \mathrm{O}_{2}{ }^{-}$and $\cdot \mathrm{OH}$ were the major species for SCP degradation. In order to verify the quenching results, EPR spectra were recorded using DMPO as the trapping agent [48], tert-butanol alcohol as -OH differentiation, and p-benzoquinone as $\cdot \mathrm{O}_{2}{ }^{-}$differentiation [49]. As displayed in Fig. 5d, when the mixed suspension was irradiated by $\mathrm{UV}$-visible light, the strong signals of spin-trapped $\cdot \mathrm{OH}$ and . $\mathrm{O}_{2}{ }^{-}$would be detected, indicating that the reactive radicals play essential roles in this aqueous system. The two radicals produced by the activation of the photocatalyst under irradiations have been well recognized to be responsible for the degradation of organics. Based on these results and analysis, the mechanism for photocatalytic degradation under UV and visible light irradiations is thus proposed and depicted in Scheme 1. It shows that the CB potential of NC-modified GCN composites is more positive than that of pure GCN. The low photocatalytic activity of GCN is mainly attributed to the weak light absorbance caused by the wide band gap. Once the GCN is loaded with nitrogen-doped carbon, the degradation rate will be highly enhanced. The outstanding photoactivity of GCN-NC(6.0) originates from the appropriate $\mathrm{N}$ content and strong coupling between GCN and NC, which provides a high concentration of active sites for fast charge carrier transport.

In general, photoactivity is largely dependent on the band edge positions of the photocatalyst. In DFT calculations, three-dimensional periodic boundary conditions were taken in the simulation. The simulation cell consists of a $2 \times 2 \mathrm{~g}_{-} \mathrm{C}_{3} \mathrm{~N}_{4}$ supercell with a vacuum width of $24 \AA$ above the graphite like GCN layer to minimize the interlayer interaction. The k-point was set to $2 \times 2 \times 1$ when optimizing structures, and all atoms were allowed to relax. The density of states (DOS) were calculated with a net k-point grid of $4 \times 4 \times 1$. An energy cutoff of $630 \mathrm{eV}$ is used. For GCN-C, adsorption energy $E_{\text {ad }}$ is determined by Eq. 3:

$E_{\mathrm{ad}}=E_{\mathrm{GCN}-\mathrm{NC}}-\left(E_{C}+E_{\mathrm{GCN}}\right)$

where $E_{\mathrm{GCN}-\mathrm{NC}}, E_{\mathrm{C}}$, and $E_{\mathrm{GCN}}$ are total energies of the GCN-C system, the isolate carbon nanosphere, and GCN layer in the same slab, respectively. DFT calculation was performed to obtain a microscopic insight on how the complex GCN-NC acts as the best transition in photocatalysis. Given the possible adsorption configuration of carbon nanosphere in the GCN sheet, the energetic favorable configuration of carbon nanosphere should be located at the hollow site of GCN layer as shown in Fig. 6a. It is noted that a hydroxyl group was attached on the outer sphere to mimic the experimental conditions. The calculated charge transfer rates between the carbon nanosphere and GCN in the most stable structures of GCN-C and GCN-NC system are $0.06 \mathrm{e}$ and $0.04 \mathrm{e}$, respectively. The distance between carbon nanosphere and GCN layer is $1.251 \AA$ with adsorption

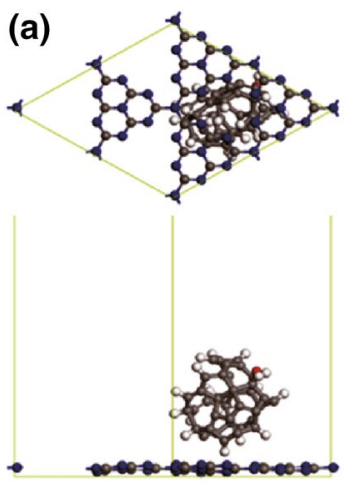

(b)

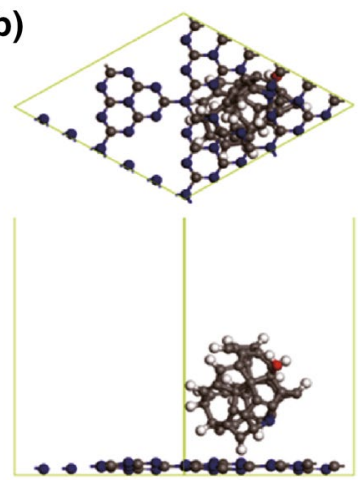

Fig. 6 a The structure of GCN-C hybrid and b GCN-NC hybrid. In the simulation, a $2 \times 2$ supercell is taken; the gray, white, blue and red balls denote carbon, hydrogen, nitrogen, and oxygen, respectively 
energy of $-0.54 \mathrm{eV}$, while those in nitrogen doping carbon nanosphere/g- $\mathrm{C}_{3} \mathrm{~N}_{4}(\mathrm{NC} / \mathrm{GCN})$ system are $1.289 \AA$ and $-1.45 \mathrm{eV}$, respectively. No chemical bond is observed in our simulation as shown in Fig. 6b, and the carbon sphere still preferred to stay at the hollow site of the GCN layer. To better understand the photocatalytic properties of the composites, the band structures of pristine GCN, GCN-C, and GCN-NC are shown in Fig. 7a-c for an in-depth analysis. Clearly, GCN has a band gap $E_{g}$ of $1.53 \mathrm{eV}$, which agrees well with other reported calculation value of $1.54 \mathrm{eV}$ using GGA functional [50]. After combining with carbon nanospheres, a remarkable decrease can be found in band gap to $0.54 \mathrm{eV}$, while the top of VB climbed to near the position of Fermi level. When introducing nitrogen into the carbon nanosphere, a further decrease in band gap can be observed, resulting in a direct band gap of $0.28 \mathrm{eV}$, with the bottom of CB dropping slightly and the top of VB nearly overlapping with the position of Fermi level, as shown in Fig. 7. To reveal the mechanism of the changes in GCN when composited with pristine carbon sphere and $\mathrm{N}$-doped carbon nanosphere, the partial density of states (PDOS) calculations of GCN-C, GCN-NC hybrids, and GCN in different systems were performed as presented in Fig. 8a-c. As can be seen, the valence band maximum (VBM) of the PDOS of the whole systems was mainly contributed by the carbon nanosphere, while GCN made the greatest contribution to the CB bottom. After N-doping, the main features of the PDOS were maintained, except for the disappearance of the peak at $-0.2 \mathrm{eV}$ in carbon nanosphere, and CB and VB of GCN changed toward the Fermi level especially. By comparing the PDOS of pristine GCN, GCN-C, and GCN-NC systems (Fig. 8c), we infer the carbon nanospheres drastically reduce the band gap of GCN as the obvious changes were observed in GCN after loading nanospheres. A slight left shift was realized after introducing $\mathrm{N}$ into carbon nanospheres, which means that the $\mathrm{CB}$ moves downward, consistent with the decrease in band gap of GCN-C material based on the band structure calculation. It is found that the carbon nanosphere is physically adsorbed on the GCN layer with van der Waals interaction. The presence of carbon nanosphere can effectively reduce the band gap of GCN, and N-doping into carbon spheres would further reduce the band gap, improving the photocatalytic performance significantly.

Based on experimental and theoretical exploration, the photocatalytic mechanism of GCN-NC can be proposed as schematically shown in Fig. 9. During the hydrothermal process, nitrogen-doped carbon nanospheres can be well integrated on GCN with strong interaction on the interface via Van der Waals force, which provide tunnels for fast interfacial electron transfer. The introduction of nitrogendoped carbon nanospheres can improve the absorption capability of visible light of pristine GCN; thus, the band gap and electronic structure have been favorably altered in the resultant catalyst composite. GCN-NC with narrow band gap can effectively absorb UV/visible light, promoting the
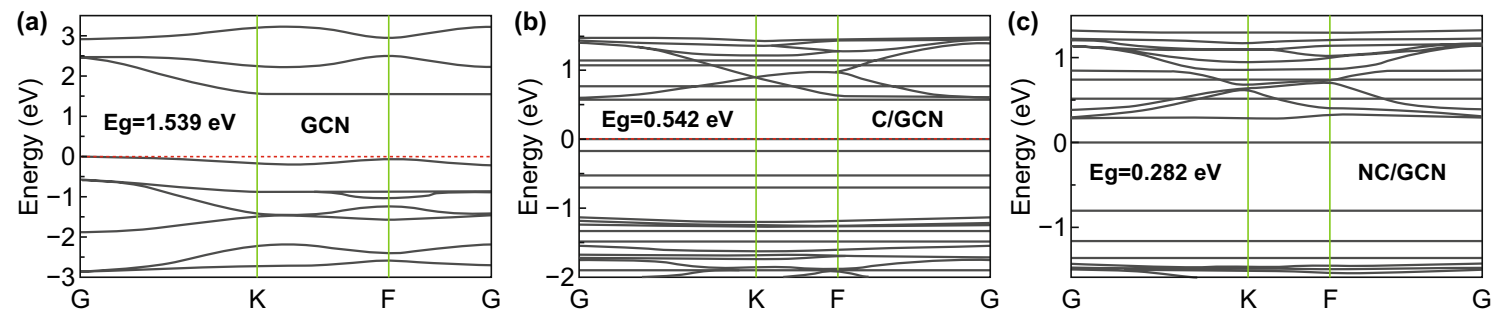

Fig. 7 a The band structures of pristine GCN, b C/GCN, and $\mathbf{c}$ NC/GCN systems; the red dash line at energy 0 is the position of Fermi level
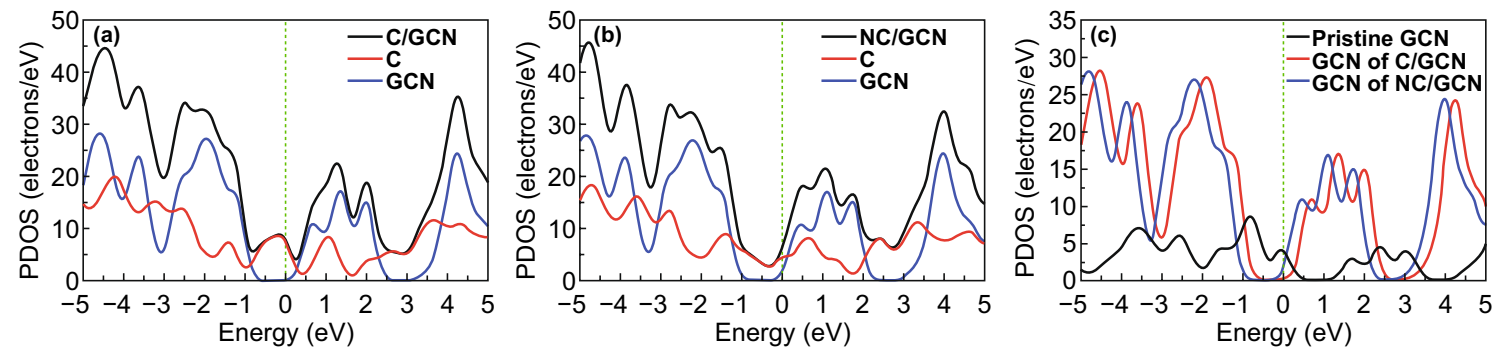

Fig. 8 a The PDOS of C/GCN, b NC/GCN systems, and $\mathbf{c}$ GCN in different systems. The vertical dash lines are the position of the Fermi level 

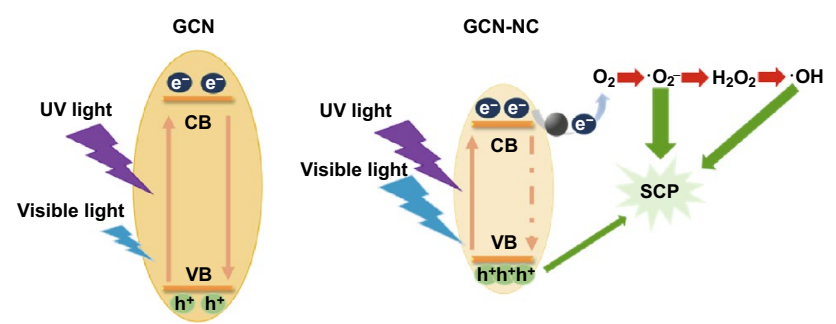

Fig. 9 Mechanism scheme of GCN-NC in photocatalytic degradation of SCP

generation of electron/hole pairs (right part of Fig. 9). Due to the strong affinity of $\mathrm{N}$ atoms, the generated electrons can be transferred by the nitrogen-doped carbon spheres more efficiently than that by pure carbon spheres, limiting the electron/hole recombination rates. Although the nitrogen doping can improve the photocatalytic performance of composite materials, the nitrogen content should be controlled in an appropriate level; otherwise, overdosage would decrease the light absorption and defer the charge transfer. As shown in Fig. 9, the photogenerated electrons inhibit the reaction of the dissolved oxygen to superoxide radical $\left(\cdot \mathrm{O}_{2}^{-}\right)$, but promote the formation of $\mathrm{H}_{2} \mathrm{O}_{2}$ at the conduction band, which further inspires the production of hydroxyl radicals $(\cdot \mathrm{OH})$. Both radicals of $\cdot \mathrm{O}_{2}{ }^{-}$and $\cdot \mathrm{OH}$ are effective to remove SCP. Based on the reported intermediates from other sulphonamides degradation study, the major oxidized products (OPs) of SCP are composed of 3-amino-6-chloropyridazine, 4-aminobenzenesulfonic acid, and hydroxylated SCP [51]. Sun et al. demonstrated that the biodegradation of the OPs is much easier compared to the parent SCP; thus, the toxicity of OPs is significantly reduced [52].

\section{Conclusions}

The atomic structure, chemical states, and electronic properties of nitrogen-doped carbon nanospheres (NC) on graphitic carbon nitride layer were characterized by TEM, XRD, FESEM, FTIR, BET, UV-Vis/DRS, XPS, EPR, and EIS techniques. The absorption edges of GCN were extended to visible light region by the coupling of $6 \mathrm{wt} \%$ nitrogendoped carbon nanospheres with a similar size about $400 \mathrm{~nm}$, while the effect of SCP degradation under visible light irradiations was affected by the nitrogen content. Among the tested samples, graphitic carbon nitride-supported carbon nanospheres doped by $6 \mathrm{wt} \%$ nitrogen exhibited the best catalytic efficiency for photodegradation of sulfachloropyridazine. Experimental results and DFT calculation imply that the carbon nanosphere is physically adsorbed on the GCN layer with van der Waals interaction. The presence of nitrogen-doped carbon nanospheres can significantly reduce the band gap of GCN, deferring the recombination rate of electron/hole pairs and thus improving the photocatalytic performance significantly. This study suggests that nitrogendoped carbon and graphitic carbon nitride composite can be applied as a new class of metal-free photocatalysts not only for wastewater treatment but also for other applications.

Acknowledgements We would like to thank the partial support from the Australian Research Council Discovery Project (No: DP170104264).

Open Access This article is licensed under a Creative Commons Attribution 4.0 International License, which permits use, sharing, adaptation, distribution and reproduction in any medium or format, as long as you give appropriate credit to the original author(s) and the source, provide a link to the Creative Commons licence, and indicate if changes were made. The images or other third party material in this article are included in the article's Creative Commons licence, unless indicated otherwise in a credit line to the material. If material is not included in the article's Creative Commons licence and your intended use is not permitted by statutory regulation or exceeds the permitted use, you will need to obtain permission directly from the copyright holder. To view a copy of this licence, visit http://creativecommons.org/licenses/by/4.0/.

Electronic supplementary material The online version of this article (https://doi.org/10.1007/s40820-019-0358-x) contains supplementary material, which is available to authorized users.

\section{References}

1. Q. Liu, X. Duan, H. Sun, Y. Wang, M.O. Tade, S. Wang, Sizetailored porous spheres of manganese oxides for catalytic oxidation via peroxymonosulfate activation. J. Phys. Chem. C 120(30), 16871-16878 (2016). https://doi.org/10.1021/acs. jpcc.6b05934

2. L. Zhou, H. Zhang, H. Sun, S. Liu, M.O. Tade, S. Wang, W. Jin, Recent advances in non-metal modification of graphitic carbon nitride for photocatalysis: a historic review. Catal. Sci. Technol. 6(19), 7002-7023 (2016). https://doi.org/10.1039/ c6cy01195k

3. K.P. Loh, Q. Bao, P.K. Ang, J. Yang, The chemistry of graphene. J. Mater. Chem. 20(12), 2277-2289 (2010). https://doi. org/10.1039/B920539J 
4. Y. Zhu, S. Murali, W. Cai, X. Li, J.W. Suk, J.R. Potts, R.S. Ruoff, Graphene and graphene oxide: synthesis, properties, and applications. Adv. Mater. 22(35), 3906-3924 (2010). https ://doi.org/10.1002/adma.201001068

5. E. Saputra, H. Zhang, Q. Liu, H. Sun, S. Wang, Egg-shaped core/shell $\alpha-\mathrm{Mn}_{2} \mathrm{O}_{3} @ \alpha-\mathrm{MnO}_{2}$ as heterogeneous catalysts for decomposition of phenolics in aqueous solutions. Chemosphere 159, 351-358 (2016). https://doi.org/10.1016/j.chemo sphere.2016.06.021

6. Y.H. Seo, M. Sung, B. Kim, Y.-K. Oh, D.Y. Kim, J.-I. Han, Ferric chloride based downstream process for microalgae based biodiesel production. Bioresour. Technol. 181, 143-147 (2015). https://doi.org/10.1016/j.biortech.2015.01.004

7. W. Liu, L. Cao, W. Cheng, Y. Cao, X. Liu et al., Frontispiece: single-site active cobalt-based photocatalyst with a long carrier lifetime for spontaneous overall water splitting. Angew. Chem. Int. Ed. 56(32), 9440-9445 (2017). https://doi. org/10.1002/anie.201783261

8. B. Ai, X. Duan, H. Sun, X. Qiu, S. Wang, Metal-free graphene-carbon nitride hybrids for photodegradation of organic pollutants in water. Catal. Today 258, 668-675 (2015). https ://doi.org/10.1016/j.cattod.2015.01.024

9. Y. Zhang, Y. Liu, W. Liu, X. Li, L. Mao, Synthesis of honeycomb-like mesoporous nitrogen-doped carbon nanospheres as Pt catalyst supports for methanol oxidation in alkaline media. Appl. Surf. Sci. 407, 64-71 (2017). https://doi.org/10.1016/j. apsusc.2017.02.158

10. R.S. Weatherup, H. Amara, R. Blume, B. Dlubak, B.C. Bayer et al., Interdependency of subsurface carbon distribution and graphene-catalyst interaction. J. Am. Chem. Soc. 136(39), 13698-13708 (2014). https://doi.org/10.1021/ja505454v

11. B.C.M. Martindale, G.A.M. Hutton, C.A. Caputo, E. Reisner, Solar hydrogen production using carbon quantum dots and a molecular nickel catalyst. J. Am. Chem. Soc. 137(18), 6018-6025 (2015). https://doi.org/10.1021/jacs.5b01650

12. X.C. Wang, K. Maeda, A. Thomas, K. Takanabe, G. Xin, J. Carlsson, K. Domen, M. Antonietti, A metal-free polymeric photocatalyst for hydrogen production from water under visible light. Nat. Mater. 8(1), 76-80 (2009). https://doi. org/10.1038/NMAT2317

13. S.C. Yan, Z.S. Li, Z.G. Zou, Photodegradation performance of $\mathrm{g}-\mathrm{C}_{3} \mathrm{~N}_{4}$ fabricated by directly heating melamine. Langmuir 25(17), 10397-10401 (2009). https://doi.org/10.1021/la900 $923 \mathrm{z}$

14. J. He, H. Sun, S. Indrawirawan, X. Duan, M. Tade, S. Wang, Novel polyoxometalate@ g- $\mathrm{C}_{3} \mathrm{~N}_{4}$ hybrid photocatalysts for degradation of dyes and phenolics. J. Colloid Interf. Sci. 456, 15-21 (2015). https://doi.org/10.1016/j.jcis.2015.06.003

15. Y. Wu, H. Wang, W. Tu, Y. Liu, S. Wu, Y.Z. Tan, J.W. Chew, Construction of hierarchical 2D-2D $\mathrm{Zn}_{3} \mathrm{In}_{2} \mathrm{~S}_{6} /$ fluorinated polymeric carbon nitride nanosheets photocatalyst for boosting photocatalytic degradation and hydrogen production performance. Appl. Catal. B 233, 58-69 (2018). https://doi. org/10.1016/j.apcatb.2018.03.105
16. Y. Zheng, J. Liu, J. Liang, M. Jaroniec, S. Qiao, Graphitic carbon nitride materials: controllable synthesis and applications in fuel cells and photocatalysis. Energy Environ. Sci. 5(5), 6717-6731 (2012). https://doi.org/10.1039/C2EE03479D

17. J. Chen, Z. Hong, Y. Chen, B. Lin, B. Gao, One-step synthesis of sulfur-doped and nitrogen-deficient $\mathrm{g}-\mathrm{C}_{3} \mathrm{~N}_{4}$ photocatalyst for enhanced hydrogen evolution under visible light. Mater. Lett. 145, 129-132 (2015). https://doi.org/10.1016/j.matle t.2015.01.073

18. P. Martín-Ramos, J. Martín-Gil, R.C. Dante, F. Vaquero, R.M. Navarro, J.L.G. Fierro, A simple approach to synthesize g- $\mathrm{C}_{3} \mathrm{~N}_{4}$ with high visible light photoactivity for hydrogen production. Int. J. Hydrogen Energy 40(23), 7273-7281 (2015). https://doi.org/10.1016/j.ijhydene.2015.04.063

19. J. Tian, Q. Liu, A.M. Asiri, K.A. Alamry, X. Sun, Ultrathin graphitic $\mathrm{C}_{3} \mathrm{~N}_{4}$ nanosheets/graphene composites: efficient organic electrocatalyst for oxygen evolution reaction. Chemsuschem 7(8), 2125-2130 (2014). https://doi.org/10.1002/ cssc. 201402118

20. Z. Sun, H. Wang, Z. Wu, L. Wang, g- $\mathrm{C}_{3} \mathrm{~N}_{4}$ based composite photocatalysts for photocatalytic $\mathrm{CO}_{2}$ reduction. Catal. Today 300, 160-172 (2017). https://doi.org/10.1016/j.catto d.2017.05.033

21. A.Y. Liu, M.L. Cohen, Structural properties and electronic structure of low-compressibility materials: $\beta-\mathrm{Si}_{3} \mathrm{~N}_{4}$ and hypothetical $\beta-\mathrm{C}_{3} \mathrm{~N}_{4}$. Phys. Rev. B 41(15), 10727-10734 (1990). https://doi.org/10.1103/PhysRevB.41.10727

22. J. Fang, H. Fan, M. Li, C. Long, Nitrogen self-doped graphitic carbon nitride as efficient visible light photocatalyst for hydrogen evolution. J. Mater. Chem. A 3(26), 13819-13826 (2015). https://doi.org/10.1039/c5ta02257f

23. S.W. Hu, L.W. Yang, Y. Tian, X.L. Wei, J.W. Ding, J.X. Zhong, P.K. Chu, Non-covalent doping of graphitic carbon nitride with ultrathin graphene oxide and molybdenum disulfide nanosheets: an effective binary heterojunction photocatalyst under visible light irradiation. J. Colloid Interf. Sci. 431, 42-49 (2014). https://doi.org/10.1016/j.jcis.2014.05.023

24. X.H. Song, L. Feng, S.L. Deng, S.Y. Xie, L.S. Zheng, Simultaneous exfoliation and modification of graphitic carbon nitride nanosheets. Adv. Mater. Interfaces 4(15), 1700339 (2017). https://doi.org/10.1002/admi.201700339

25. G. Zhang, M. Zhang, X. Ye, X. Qiu, S. Lin, X. Wang, Iodine modified carbon nitride semiconductors as visible light photocatalysts for hydrogen evolution. Adv. Mater. 26(5), 805-809 (2014). https://doi.org/10.1002/adma.201303611

26. Z. Lin, X. Wang, Nanostructure engineering and doping of conjugated carbon nitride semiconductors for hydrogen photosynthesis. Angew. Chem. Int. Ed. 52(6), 1735-1738 (2013). https://doi.org/10.1002/anie.201209017

27. H. Sun, X. Zhou, H. Zhang, W. Tu, An efficient exfoliation method to obtain graphitic carbon nitride nanosheets with superior visible-light photocatalytic activity. Int. J. Hydrogen Energy 42(12), 7930-7937 (2017). https://doi.org/10.1016/j. ijhydene.2016.12.080 
28. S. Hu, W. Zhang, J. Bai, G. Lu, L. Zhang, G. Wu, Construction of a $2 \mathrm{D} / 2 \mathrm{D}$ g- $\mathrm{C}_{3} \mathrm{~N}_{4} / \mathrm{rGO}$ hybrid heterojunction catalyst with outstanding charge separation ability and nitrogen photofixation performance via a surface protonation process. RSC Adv. 6(31), 25695-25702 (2016). https://doi.org/10.1039/c5ra2 $8123 \mathrm{~g}$

29. R.C. Pawar, S. Kang, S.H. Ahn, C.S. Lee, Gold nanoparticle modified graphitic carbon nitride/multi-walled carbon nanotube $\left(\mathrm{g}-\mathrm{C}_{3} \mathrm{~N}_{4} / \mathrm{CNTs} / \mathrm{Au}\right)$ hybrid photocatalysts for effective water splitting and degradation. RSC Adv. 5(31), 2428124292 (2015). https://doi.org/10.1039/c4ra15560b

30. G. Rajender, B. Choudhury, P.K. Giri, In situ decoration of plasmonic au nanoparticles on graphene quantum dots-graphitic carbon nitride hybrid and evaluation of its visible light photocatalytic performance. Nanotechnology 28(39), 395703 (2017). https://doi.org/10.1088/1361-6528/aa810a

31. Y. Sun, C. Li, Y. Xu, H. Bai, Z. Yao, G. Shi, Chemically converted graphene as substrate for immobilizing and enhancing the activity of a polymeric catalyst. Chem. Commun. 46(26), 4740-4742 (2010). https://doi.org/10.1039/C001635G

32. S. Liu, J. Ke, H. Sun, J. Liu, M.O. Tade, S. Wang, Size dependence of uniformed carbon spheres in promoting graphitic carbon nitride toward enhanced photocatalysis. Appl. Catal. B 204, 358-364 (2017). https://doi.org/10.1016/j.apcat b. 2016.11 .048

33. G. Dong, K. Zhao, L. Zhang, Carbon self-doping induced high electronic conductivity and photoreactivity of $\mathrm{g}-\mathrm{C}_{3} \mathrm{~N}_{4}$. Chem. Commun. 48(49), 6178-6180 (2012). https://doi.org/10.1039/ C2CC32181E

34. D. Gao, Y. Liu, P. Liu, M. Si, D. Xue, Atomically thin B doped $\mathrm{g}_{-} \mathrm{C}_{3} \mathrm{~N}_{4}$ nanosheets: high-temperature ferromagnetism and calculated half-metallicity. Sci. Rep. 6, 35768 (2016). https://doi. org/10.1038/srep35768

35. J. Cao, X. Yin, L. Wang, M. Guo, J. Xu, Z. Chen, Enhanced electrocatalytic activity of platinum nanoparticles supported on nitrogen-modified mesoporous carbons for methanol electrooxidation. Int. J. Hydrogen Energy 40(7), 2971-2978 (2015). https://doi.org/10.1016/j.ijhydene.2015.01.014

36. J. Liu, Y. Song, H. Xu, J. Zhu, J. Lian et al., Non-metal photocatalyst nitrogen-doped carbon nanotubes modified $m p g-\mathrm{C}_{3} \mathrm{~N}_{4}$ : facile synthesis and the enhanced visible-light photocatalytic activity. J. Colloid Interf. Sci. 494, 38-46 (2017). https://doi. org/10.1016/j.jcis.2017.01.010

37. L. Jia, D.H. Wang, Y.X. Huang, A.W. Xu, H.Q. Yu, Highly durable $\mathrm{N}$-doped graphene/CdS nanocomposites with enhanced photocatalytic hydrogen evolution from water under visible light irradiation. J. Phys. Chem. C 115(23), 1146611473 (2011). https://doi.org/10.1021/jp2023617

38. G. Wang, Y. Sun, D. Li, H.W. Liang, R. Dong, X. Feng, K. Müllen, Controlled synthesis of $\mathrm{N}$-doped carbon nanospheres with tailored mesopores through self-assembly of colloidal silica. Angew. Chem. Int. Ed. 54(50), 15191-15196 (2015). https://doi.org/10.1002/anie.201507735
39. J. Clark Stewart, D. Segall Matthew, J. Pickard Chris, J. Hasnip Phil, I.J. Probert Matt, K. Refson, C. Payne Mike, First principles methods using CASTEP. Z. Kristall.-Cryst. Mater. 220(00), 567-570 (2005)

40. J.P. Perdew, A. Ruzsinszky, G.I. Csonka, O.A. Vydrov, G.E. Scuseria, L.A. Constantin, X. Zhou, K. Burke, Restoring the density-gradient expansion for exchange in solids and surfaces. Phys. Rev. Lett. 100(13), 136406 (2008). https://doi. org/10.1103/PhysRevLett.100.136406

41. A. Tkatchenko, M. Scheffler, Accurate molecular van der waals interactions from ground-state electron density and freeatom reference data. Phys. Rev. Lett. 102(7), 073005 (2009). https://doi.org/10.1103/PhysRevLett.102.073005

42. Y. Zhou, L. Zhang, W. Huang, Q. Kong, X. Fan, M. Wang, J. Shi, N-doped graphitic carbonincorporated $g-\mathrm{C}_{3} \mathrm{~N}_{4}$ for remarkably enhanced photocatalytic $\mathrm{H}_{2}$ evolution under visible light. Carbon 99, 111-117 (2016). https://doi.org/10.1016/j.carbo n.2015.12.008

43. C. Han, Y. Wang, Y. Lei, B. Wang, N. Wu, Q. Shi, Q. Li, In situ synthesis of graphitic- $\mathrm{C}_{3} \mathrm{~N}_{4}$ nanosheet hybridized $\mathrm{N}$-doped $\mathrm{TiO}_{2}$ nanofibers for efficient photocatalytic $\mathrm{H}_{2}$ production and degradation. Nano Res. 8(4), 1199-1209 (2015). https://doi.org/10.1007/s12274-014-0600-2

44. P. Tiong, H.O. Lintang, S. Endud, L. Yuliati, Improved interfacial charge transfer and visible light activity of reduced graphene oxide-graphitic carbon nitride photocatalysts. RSC Adv. 5(114), 94029-94039 (2015). https://doi.org/10.1039/ c5ra17967j

45. H. Wang, Y. Wu, M.B. Feng, W.G. Tu, T. Xiao et al., Visible-light-driven removal of tetracycline antibiotics and reclamation of hydrogen energy from natural water matrices and wastewater by polymeric carbon nitride foam. Water Res. 144, 215-225 (2018). https://doi.org/10.1016/j.watres.2018.07.025

46. M. Conde-Cid, D. Fernández-Calviño, J.C. Nóvoa-Muñoz, M. Arias-Estévez, M. Díaz-Raviña, A. Núñez-Delgado, M.J. Fernández-Sanjurjo, E. Álvarez-Rodríguez, Degradation of sulfadiazine, sulfachloropyridazine and sulfamethazine in aqueous media. J. Environ. Manage. 228, 239-248 (2018). https://doi.org/10.1016/j.jenvman.2018.09.025

47. Y. Wu, H. Wang, W. Tu, S. Wu, J.W. Chew, Effects of composition faults in ternary metal chalcogenides $\left(\mathrm{Zn}_{\mathrm{x}} \mathrm{In}_{2} \mathrm{~S}_{3+\mathrm{x}}, \mathrm{x}=1-5\right)$ layered crystals for visible-light-driven catalytic hydrogen generation and carbon dioxide reduction. Appl. Catal. B 256, 117810 (2019). https://doi.org/10.1016/j.apcatb.2019.117810

48. L.Y. Zang, K. Stone, W.A. Pryor, Detection of free radicals in aqueous extracts of cigarette tar by electron spin resonance. Free Radical Biol. Med. 19(2), 161-167 (1995). https://doi. org/10.1016/0891-5849(94)00236-D

49. G.V. Buxton, C.L. Greenstock, W.P. Helman, A.B. Ross, Critical review of rate constants for reactions of hydrated electrons, hydrogen atoms and hydroxyl radicals $\left(\cdot \mathrm{OH} / \cdot \mathrm{O}^{-}\right.$in aqueous solution. J. Phys. Chem. Ref. Data 17(2), 513-886 (1988). https://doi.org/10.1063/1.555805 
50. B. Jing, Z. Ao, Z. Teng, C. Wang, J. Yi, T. An, Density functional theory study on the effects of oxygen groups on band gap tuning of graphitic carbon nitrides for possible photocatalytic applications. Sustainable Mater. Technol. 16, 12-22 (2018). https://doi.org/10.1016/j.susmat.2018.04.001

51. L.H. Hu, P.M. Flanders, P.L. Miller, T.J. Strathmann, Oxidation of sulfamethoxazole and related antimicrobial agents by
$\mathrm{TiO}_{2}$ photocatalysis. Water Res. 41, 2612-2626 (2007). https ://doi.org/10.1016/j.watres.2007.02.026

52. X.H. Sun, M.B. Feng, S.Y. Dong, Y. Qi, L. Sun, N. Nesnas, Vk Sharma, Removal of sulfachloropyridazine by ferrate (VI): kinetics reaction pathways biodegradation and toxicity evaluation. Chem. Eng. J. 372, 742-751 (2019). https://doi. org/10.1016/j.cej.2019.04.121 